\title{
Telmisartan inhibits AGE-induced C-reactive protein production through downregulation of the receptor for AGE via peroxisome proliferator-activated receptor-gamma activation
}

\author{
T. Yoshida $\cdot$ S. Yamagishi $\cdot$ K. Nakamura $\cdot$ T. Matsui • \\ T. Imaizumi • M. Takeuchi $\cdot$ H. Koga $・$ T. Ueno $・$ M. Sata
}

Received: 20 April 2006 / Accepted: 3 August 2006 / Published online: 27 September 2006

(C) Springer-Verlag 2006

\begin{abstract}
Aims/hypothesis C-reactive protein (CRP), an acute-phase reactant produced mainly by the liver, is elevated in diabetes, thus contributing to the development and progression of atherosclerosis. However, the molecular mechanism underlying the elevation of CRP in diabetes is not fully understood. Since a crosstalk between AGE and angiotensin II (Ang II) has been proposed in the pathogenesis of accelerated atherosclerosis in diabetes, we examined here whether and how telmisartan, a unique Ang II type 1 receptor blocker (ARB) with peroxisome proliferatoractivated receptor- $\gamma$ (PPAR- $\gamma$ )-modulating activity, could inhibit AGE-induced CRP expression in a human hepatoma cell line, Hep3B cells.

Methods Protein levels of the receptor for AGE (RAGE) were analysed by western blots. Gene expression was analysed by quantitative real-time RT-PCR. CRP released into the medium was measured with ELISA. Intracellular formation of reactive oxygen species (ROS) was measured using the fluorescent probe CM- $\mathrm{H}_{2}$ DCFDA.

Results Telmisartan, but not candesartan, another ARB, downregulated $R A G E$ mRNA levels in a dose-dependent manner. Telmisartan decreased basal as well as AGE-
\end{abstract}

T. Yoshida $\cdot$ S. Yamagishi $(\bowtie) \cdot K$. Nakamura $\cdot$ T. Matsui $\cdot$

T. Imaizumi $\cdot$ H. Koga $\cdot$ T. Ueno $\cdot$ M. Sata

Department of Medicine, Kurume University School of Medicine,

67 Asahi-machi,

Kurume 830-0011, Japan

e-mail: shoichi@med.kurume-u.ac.jp

M. Takeuchi

Department of Pathophysiological Science,

Faculty of Pharmaceutical Science, Hokuriku University,

Kanazawa, Japan induced RAGE protein expression in Hep3B cells. Furthermore, telmisartan dose-dependently inhibited AGE-induced ROS generation and subsequent $C R P$ gene and protein induction in Hep3B cells. GW9662, an inhibitor of PPAR- $\gamma$, blocked the inhibitory effects of telmisartan on RAGE expression and its downstream signalling in Hep3B cells.

Conclusions/interpretation Our present study indicates a unique beneficial aspect of telmisartan: it may work as an anti-inflammatory agent against AGE by suppressing RAGE expression via PPAR- $\gamma$ activation in the liver and may play a protective role in vascular injury in diabetes.

Keywords AGE - Macrovascular disease - Oxidative stress
Abbreviations
$\mathrm{Ab} \quad$ antibody
Ang II angiotensin II
ARB Ang II type 1 receptor blocker
CRP C-reactive protein
GAPDH glyceraldehyde-3-phosphate dehydrogenase
PPAR- $\gamma$ peroxisome proliferator-activated receptor- $\gamma$
RAGE receptor for AGE
ROS reactive oxygen species

\section{Introduction}

Non-enzymatic modification of proteins by reducing sugars, a process that is also known as the Maillard reaction, progresses at an extremely accelerated rate in diabetes, thus leading to the formation of AGE [1]. Recent understanding of this process has revealed that the receptor 
system for AGE (RAGE) plays a central role in the pathogenesis of diabetic vascular complications such as cardiovascular disease [1].

C-reactive protein (CRP) is an acute-phase reactant mainly produced by the liver in response to pro-inflammatory cytokines [2]. Numerous studies have shown that CRP is one of the most powerful predictors for future cardiovascular events [2]. In addition, CRP elicits endothelial dysfunction, pro-inflammatory reactions and vascular smooth muscle cell proliferation, thereby directly contributing to the development and progression of atherosclerosis [3]. However, the molecular mechanism for the elevation of CRP in diabetes is not fully understood. Since crosstalk between the AGE-RAGE system and angiotensin II (Ang II) has been proposed in the pathogenesis of accelerated atherosclerosis in diabetes [1], we examined here whether and how telmisartan, a unique Ang II type 1 receptor blocker (ARB) with peroxisome proliferator-activated receptor- $\gamma$ (PPAR- $\gamma$ )-modulating activity [4], could inhibit AGE-induced CRP production in a human hepatoma cell line, Hep3B cells.

\section{Materials and methods}

Materials

Telmisartan and candesartan were generously given by Boehringer Ingelheim (Ingelheim, Germany) and Sankyo Co. Ltd. (Tokyo, Japan), respectively. Troglitazone, ciglitazone and GW9662, an inhibitor of PPAR- $\gamma$ were purchased from Cayman Chemical (Ann Arbor, MI, USA). Antibody (Ab) directed against PPAR- $\gamma$ was from Santa Cruz Biotechnology Inc. (Santa Cruz, CA, USA). BSA (fraction $\mathrm{V})$, polymyxin B sulphate, rabbit non-specific serum and curcumin were from Sigma (St Louis, MO, USA). Abs against RAGE and glyceraldehyde-3-phosphate dehydrogenase (GAPDH) were purchased from Cell Signaling (Beverly, MA, USA). Rabbit non-specific IgG was from Santa Cruz. IL-6 was from PeproTech Inc. (Rocky Hill, NJ, USA).

\section{Preparation of AGE-BSA}

AGE-BSA was prepared as described previously [5]. Briefly, BSA was incubated under sterile conditions with D-glyceraldehyde for 7 days. Then unincorporated sugars were removed by dialysis against PBS. Control nonglycated BSA was incubated in the same conditions except for the absence of reducing sugar. Preparations were tested for endotoxin using an Endospecy ES-20S system (Seikagaku Co., Tokyo, Japan); no endotoxin was detected.
Preparation of Abs directed against AGE-BSA

Polyclonal Abs directed against AGE-BSA were prepared as described previously [6].

Preparation of antiserum directed against RAGE

Antiserum directed against human RAGE for neutralising assays was prepared as described previously [6].

Cells

Human hepatoma Hep3B cells were obtained from ATCC (Manassas, VA, USA) and maintained in DMEM supplemented with $10 \%$ fetal bovine serum.

Quantitative real-time RT-PCR

Hep3B cells were treated with or without $100 \mu \mathrm{g} / \mathrm{ml} \mathrm{BSA}$, $100 \mu \mathrm{g} / \mathrm{ml} \mathrm{AGE-BSA}$ or $10 \mathrm{ng} / \mathrm{ml} \mathrm{IL-6}$ in the presence or absence of telmisartan for $24 \mathrm{~h}$. Then total RNA was extracted with Trizol Reagent (Invitrogen, Carlsbad, CA, USA) according to the manufacturer's instructions. Quantitative real-time RT-PCR was performed using SYBR green reagent (Applied Biosystems, Foster City, CA, USA) according to the manufacturer's recommendation. Primers for CRP and GAPDH were as follows: CRP forward primer, 5'-CCCTGAACTTTCAGCCGAATACA3'; CRP reverse primer 5'-CGTCCTGCTGCCAGTGA TACA-3', GAPDH forward primer, 5'-TGCACCACCAA CTGCTTAGC-3'; GAPDH reverse primer 5'-GGCATGG ACTGTGGTCATGAG-3'. The forward and reverse primers used for RAGE mRNA detection were 5'-GCTGGAATGG AAACTGAACACAGG-3' and 5'-TTCCCAGGAATCTGG TAGACACG-3', respectively.

Western blot analysis

Proteins were extracted from Hep3B cells with lysis buffer, and then separated by SDS-PAGE and transferred to nitrocellulose membranes as described previously [5]. Immune complexes were visualised with an enhanced chemiluminescence detection system (Amersham Bioscience, Amersham, Bucks, UK).

Measurement of intracellular reactive oxygen species generation

Hep3B cells were treated with $100 \mu \mathrm{g} / \mathrm{ml}$ BSA or AGEBSA in the presence or absence of telmisartan for $24 \mathrm{~h}$. Then the intracellular formation of reactive oxygen species (ROS) was measured using the fluorescent probe CM$\mathrm{H}_{2}$ DCFDA (Molecular Probes, Eugene, OR, USA) [5]. 
Measurement of CRP

Hep3B cells were treated with $100 \mu \mathrm{g} / \mathrm{ml}$ BSA or AGEBSA in the presence or absence of telmisartan for $48 \mathrm{~h}$. CRP contents in the medium were measured with a CRP ELISA kit (Assay Pro, Winfield, MO, USA) according to the manufacturer's instructions.

\section{Statistical analysis}

All values were presented as means \pm SEM. Statistical significance was evaluated using Student's $t$ test for paired comparisons; $p<0.05$ was considered significant. The experiments were performed in triplicate or quadruplicate. We performed the same experiments three times.

\section{Results}

Effects of telmisartan on RAGE expression in Hep3B cells

RAGE is a signal-transducing receptor for AGE [1]. Therefore, we first examined the effect of telmisartan on RAGE expression in Hep3B cells. Telmisartan, but not another ARB, candesartan, dose-dependently decreased $R A G E$ mRNA levels in Hep3B cells, which was blocked by GW9662, an inhibitor of PPAR- $\gamma$ (Fig. 1a). In addition, troglitazone and ciglitazone, full agonists of PPAR- $\gamma$, modestly, but significantly, suppressed $R A G E$ mRNA levels in Hep3B cells, while GW9662 alone increased them. Moreover, telmisartan decreased RAGE protein levels in non-glycated BSA- or AGE-BSA-exposed Hep3B cells,

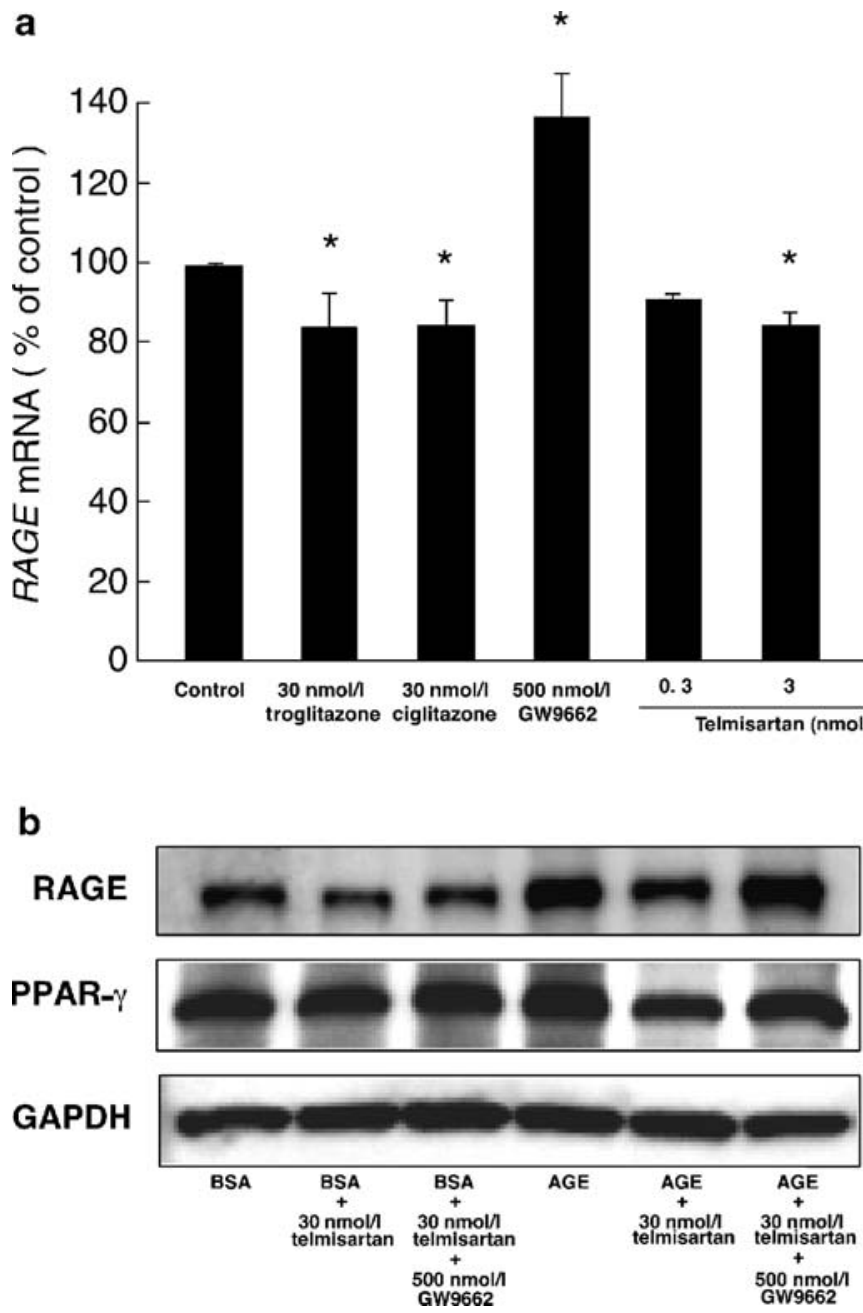

Fig. 1 Effects of telmisartan on RAGE expression in Hep3B cells. a Relative gene expression levels of RAGE are shown. ${ }^{*} p<0.05$ compared with control. \#\#p<0.01 compared with $30 \mathrm{nmol} / 1$ telmisartan alone. b Hep3B cells were treated with $100 \mu \mathrm{g} / \mathrm{ml} \mathrm{BSA}$ or AGE-BSA in the presence or absence of $30 \mathrm{nmol} / 1$ telmisartan or $500 \mathrm{nmol} / 1 \mathrm{GW} 9662$ for $24 \mathrm{~h}$. Then, RAGE and PPAR- $\gamma$ protein levels were analysed by

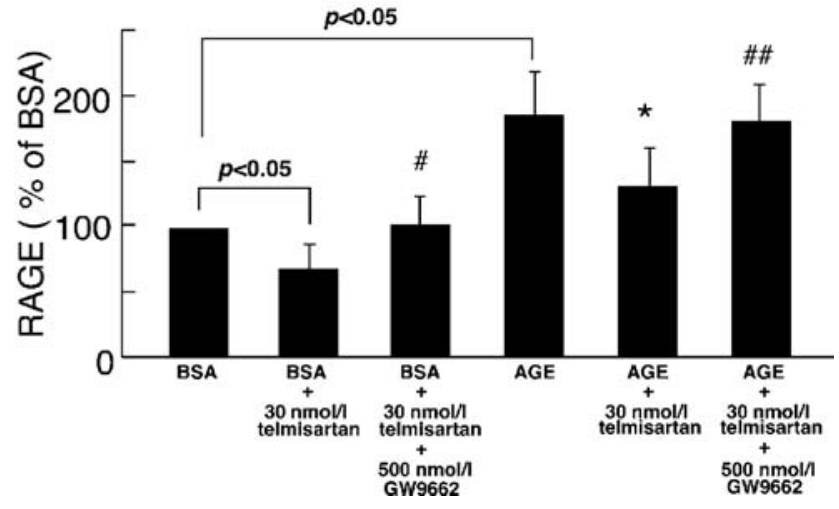

western blots. The graph shows relative RAGE protein levels. Data were normalised with respect to the intensity of GAPDH signals and related to the value with BSA alone. ${ }^{*} p<0.05$ compared with AGE alone. $\# p<0.05$ compared with BSA plus $30 \mathrm{nmol} / 1$ telmisartan. \#\#p<0.05 compared with AGE plus $30 \mathrm{nmol} / 1$ telmisartan. The experiments were performed in triplicate. Values are means \pm SEM from a representative test 
which was prevented by GW9662 (Fig. 1b). We confirmed that telmisartan at $30 \mathrm{nmol} / 1$ for $48 \mathrm{~h}$ did not affect cell viability with a dye exclusion method (data not shown). Further, western blot analysis revealed that PPAR- $\gamma$ was expressed in Hep3B cells (Fig. 1b).

Effects of telmisartan on AGE-elicited ROS generation in Hep3B cells

We next examined whether telmisartan could inhibit the downstream signalling induced by AGE-RAGE. For this, we studied the effect of telmisartan on intracellular ROS generation in AGE-exposed Hep3B cells. As shown in
Fig. 2a, telmisartan, but not candesartan, decreased the AGE-elicited ROS generation in a dose-dependent manner, which was blocked by GW9662. Polyclonal Abs $(10 \mu \mathrm{g} / \mathrm{ml})$ directed against AGE or $0.1 \%$ antiserum against RAGE, but not rabbit non-specific IgG Abs or serum, completely blocked the AGE-induced ROS generation (Fig. 2a). In addition, troglitazone and ciglitazone modestly, but significantly, inhibited the ROS generation in AGE-exposed Hep3B cells. Since pre-incubation of AGE-containing media with $1 \mu \mathrm{g} / \mathrm{ml}$ polymyxin $\mathrm{B}$, an inhibitor of endotoxin, for $3 \mathrm{~min}$ did not decrease the ROS generation in AGE-exposed Hep3B cells, it is unlikely that the effects of AGE are simply mediated by endotoxin.
Fig. 2 Effects of telmisartan on ROS generation (a) and CRP expression (b-d) in Hep3B cells. a ROS generation related to the value with BSA alone. b, c $C R P$ gene expression was analysed by quantitative realtime RT-PCR. Relative gene expression levels of $C R P$ are shown. d CRP levels in the medium were measured by ELISA. ${ }^{*} p<0.05$ compared with $100 \mu \mathrm{g} / \mathrm{ml}$ AGE alone. \#\#p<0.05 compared with AGE plus $30 \mathrm{nmol} / 1$ telmisartan. $\# p<0.05$ compared with $10 \mathrm{ng} / \mathrm{ml}$ IL-6 alone. The experiments were performed in triplicate or quadruplicate. Values are means \pm SEM from a representative test
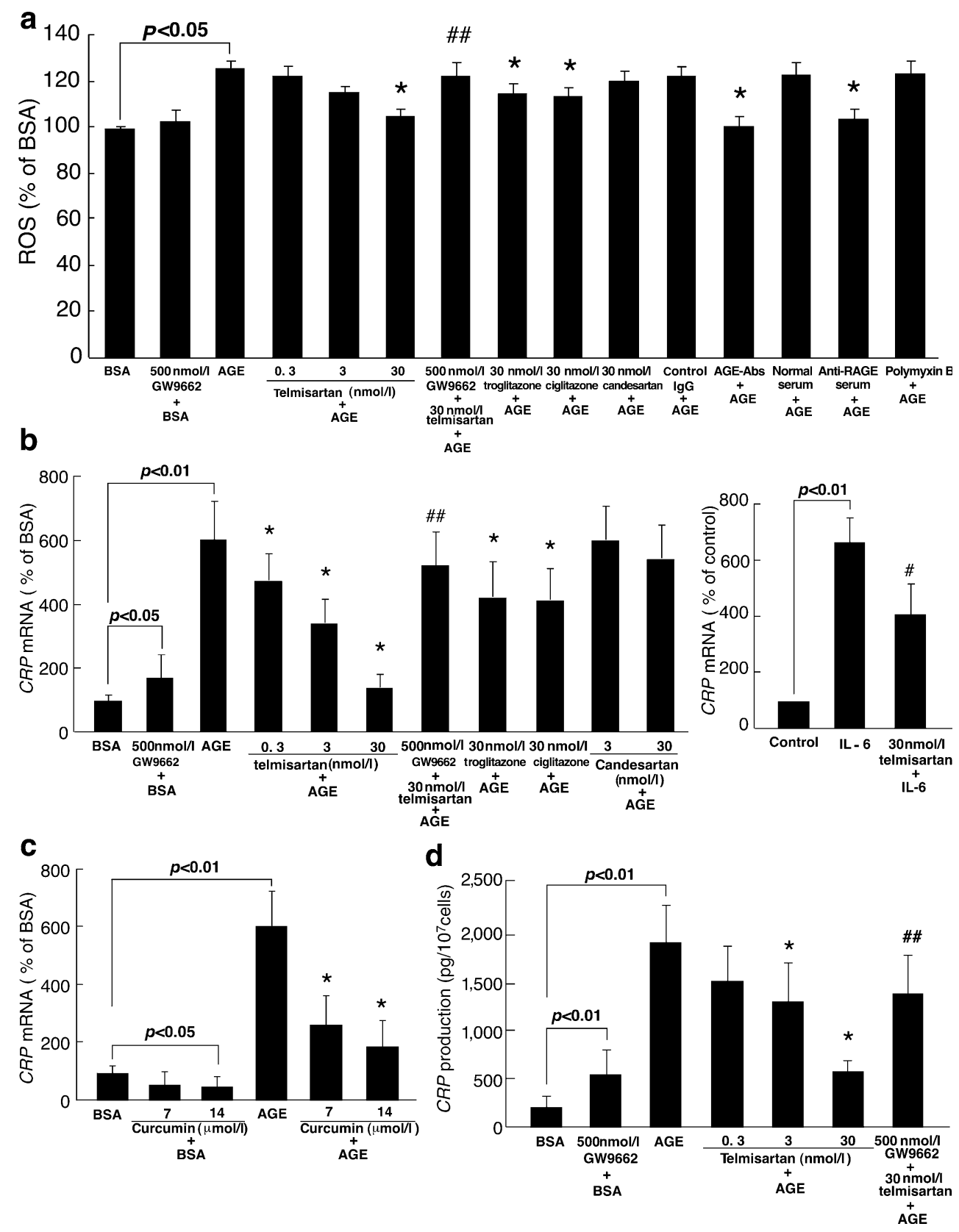
Effects of telmisartan on AGE-induced CRP gene expression in Hep3B cells

We further investigated whether telmisartan could inhibit the AGE-induced CRP expression in Hep3B cells. As shown in Fig. 2b, quantitative real-time RT-PCR analysis revealed that telmisartan, but not candesartan, dose-dependently decreased CRP mRNA levels in AGE-exposed Hep3B cells. GW9662 blocked the inhibitory effects of telmisartan on CRP mRNA levels. Further, troglitazone and ciglitazone modestly, but significantly, inhibited the AGE-induced upregulation of $C R P$ mRNA levels. An antioxidant, $N$-acetylcysteine, a glutathione peroxidase mimic, ebselen, or an inhibitor of NADPH oxidase, diphenylene iodonium, blocked the AGE-induced upregulation of $C R P$ mRNA levels in Hep3B cells as well (results not shown). These observations suggest that telmisartan could suppress the AGE-induced CRP gene induction in Hep3B cells through its antioxidative property via PPAR- $\gamma$ activation. Although PPAR- $\gamma$ full agonists had similar effects to telmisartan, partial agonist-specific effects may be involved in telmisartan's action because the magnitude of the effects of full agonists was lower than those of telmisartan. Further, since GW9662 alone slightly increased CRP mRNA levels, PPAR- $\gamma$ may also regulate basal $C R P$ gene expression in Hep3B cells. Telmisartan inhibited IL-6-induced CRP gene expression in Hep3B cells as well.

To elucidate the underlying mechanism for AGE-induced $C R P$ gene induction, we investigated the involvement of the redox-sensitive transcriptional factor nuclear factor- $\mathrm{KB}$ $(\mathrm{NF}-\mathrm{KB})$ in AGE signalling. AGE increased NF- $\mathrm{BB}$ promoter activity in Hep3B cells by about 1.4-fold. Furthermore, curcumin, an inhibitor of NF-KB, decreased CRP mRNA levels in AGE-exposed Hep3B cells in a dose-dependent manner (Fig. 2c). These observations suggest that the AGERAGE-mediated ROS generation may induce CRP gene expression through the transcriptional activation of NF- $\mathrm{KB}$.

Effects of telmisartan on AGE-induced CRP protein expression in Hep3B cells

We next investigated whether telmisartan could inhibit the AGE-induced CRP expression in Hep3B cells. As shown in Fig. 2d, telmisartan dose-dependently inhibited the production of CRP by AGE-exposed Hep3B cells, which was blocked by GW9662. As in the case of CRP mRNA levels, GW9662 alone slightly, but significantly, increased CRP production in Hep3B cells.

\section{Discussion}

Recently, telmisartan has been shown to have a unique property that activates PPAR- $\gamma[4,7]$. Since PPAR- $\gamma$ influences gene expression involved in carbohydrate and lipid metabolism, and since ligands for PPAR- $\gamma$ can improve insulin sensitivity, telmisartan could be a promising 'cardiometabolic sartan' that targets both diabetes and cardiovascular disease in patients with hypertension [7]. Indeed, a recent clinical report has shown that replacement of valsartan and candesartan by telmisartan improves insulin resistance and decreases CRP levels in hypertensive patients with type 2 diabetes [8]. In this study, we have extended these previous findings. We demonstrated here for the first time that $30 \mathrm{nmol} / 1$ telmisartan, but not candesartan, could block the AGE-elicited CRP induction in Hep3B cells by suppressing RAGE expression and subsequent ROS generation via PPAR- $\gamma$ activation. Since $30 \mathrm{nmol} / \mathrm{l}$ ARBs cannot efficiently block the Ang II binding to type 1 receptor [5] and GW9662, an inhibitor of PPAR- $\gamma$, blocked the inhibitory effects of telmisartan on RAGE expression and its downstream signalling in Hep3B cells, our present study suggests a unique beneficial aspect of telmisartan - it may work as an anti-inflammatory agent against AGE by suppressing RAGE expression via PPAR- $\gamma$ activation in the liver and may play a protective role in vascular injury in diabetes. The observation that telmisartan inhibited $C R P$ gene expression in IL-6-exposed Hep3B cells (Fig. 2b) was also consistent with the previous finding that PPAR- $\gamma$ can function as a negative modulator of IL-6 signalling [9].

Recently, PPAR- $\gamma$ agonists have been reported to downregulate RAGE expression in endothelial cells and subsequently inhibit AGE-induced monocyte chemoattractant protein-1 expression, thus limiting the cells' susceptibility to pro-inflammatory AGE effects [10]. Telmisartan could directly modulate the development of vascular dysfunction in diabetic patients due to its unique PPAR- $\gamma$ modulating activity.

Acknowledgement This work was supported in part by Grants of Collaboration with Venture Companies Project from the Ministry of Education, Culture, Sports, Science and Technology, Japan (S. Yamagishi).

Duality of interest There is no duality of interest.

\section{References}

1. Yamagishi S, Imaizumi T (2005) Diabetic vascular complications: pathophysiology, biochemical basis and potential therapeutic strategy. Curr Pharm Des 11:2279-2299

2. Ridker PM, Hennekens CH, Buring JE, Rifai N (2000) $\mathrm{C}$-reactive protein and other markers of inflammation in the prediction of cardiovascular disease in women. N Engl J Med 342:836-843

3. Jialal I, Devaraj S, Venugopal SK (2004) C-reactive protein: risk marker or mediator in atherothrombosis? Hypertension 44:6-11

4. Benson SC, Pershadsingh HA, Ho CI et al (2004) Identification of telmisartan as a unique angiotensin II receptor antagonist 
with selective PPARgamma-modulating activity. Hypertension 43:993-1002

5. Yamagishi S, Takeuchi M, Matsui T, Nakamura K, Imaizumi T, Inoue H (2005) Angiotensin II augments advanced glycation end product-induced pericyte apoptosis through RAGE overexpression. FEBS Lett 579:4265-4270

6. Fukami K, Ueda S, Yamagishi S et al (2004) AGEs activate mesangial TGF- $\beta$-Smad signaling via an angiotensin II type 1 receptor interaction. Kidney Int 66:2137-2147

7. Schupp M, Clemenz M, Gineste R et al (2005) Molecular characterization of new selective peroxisome proliferators-activat- ed receptor $\gamma$ modulators with angiotensin receptor blocking activity. Diabetes 54:3442-3452

8. Miura Y, Yamamoto N, Tsunekawa S et al (2005) Replacement of valsartan and candesartan by telmisartan in hypertensive patients with type 2 diabetes. Diabetes Care 28:757-758

9. Wang LH, Yang XY, Zhang X, Farrar WL (2005) Nuclear receptors as negative modulators of STAT3 in multiple myeloma. Cell Cycle 4:242-245

10. Marx N, Walcher D, Ivanova N et al (2004) Thiazolidinediones reduce endothelial expression of receptors for advanced glycation end products. Diabetes 53:2662-2668 